Revue internationale de l'économie sociale

Recma

\title{
Banques coopératives : de l'idéal solidaire à la réalité des
}

salaires

\section{Gilles Caire, Pascal Glémain et Sophie Nivoix}

Numéro 339, janvier 2016

URI : https://id.erudit.org/iderudit/1035590ar

DOI : https://doi.org/10.7202/1035590ar

Aller au sommaire du numéro

\section{Éditeur(s)}

Association Recma

\section{ISSN}

1626-1682 (imprimé)

2261-2599 (numérique)

Découvrir la revue

\section{Citer cet article}

Caire, G., Glémain, P. \& Nivoix, S. (2016). Banques coopératives : de l'idéal solidaire à la réalité des salaires. Revue internationale de l'économie sociale, (339), 101-111. https://doi.org/10.7202/1035590ar 


\section{BANQUES COOPÉRATIVES: DE L'IDÉAL SOLIDAIRE À LA RÉALITÉ DES SALAIRES}

\author{
par Gilles Caire*, Pascal Glémain** et Sophie Nivoix***
}

Interroger les rémunérations dans l'économie sociale et solidaire (ESS) en général est une question sensible. Cela l'est tout autant dans le secteur bancaire, dont l'image n'est pas des plus positives depuis la crise dite des subprimes de 2008. Traiter des rémunérations au sein des banques coopératives devient dès lors une entreprise doublement délicate. De plus, si la question de l'emploi dans l'ESS et de sa qualité a déjà été abordée par les travaux de Richez-Battesti et alii $^{(1)}$, il n'existe pas à notre connaissance d'état des lieux des rémunérations au sein des groupes bancaires coopératifs.

Dans le cadre de cet article, nous proposons une première analyse comparative entre les banques coopératives et les banques capitalistes ${ }^{(2)}$. A la question " les salariés des établissements bancaires coopératifs sont-ils plus ou moins bien rémunérés que ceux qui travaillent dans les établissements bancaires commerciaux?", nous tentons d'apporter une réponse de la façon la plus objective possible, en nous appuyant sur les données des documents de référence publics (rapports d'activité, rapports financiers, rapports RSE) et des bilans sociaux (lorsqu'ils sont disponibles) des cinq grands groupes bancaires privés français coopératifs ou commerciaux: Crédit agricole-LCL (CA), BPCE, Crédit mutuelCIC (CM), BNP Paribas (BNPP) et Société générale (SG).

Après un bref rappel de l'état des connaissances quant aux rémunérations dans l'économie sociale reposant sur l'analyse des déclarations annuelles de données sociales (DADS), nous observons les rémunérations moyennes dans les banques, puis les inégalités qui transparaissent au travers des grilles salariales. Ensuite, nous nous intéressons aux rémunérations hors salaire, à savoir la participation, l'intéressement, voire l'abondement lorsqu'il était mentionné. Enfin, il nous a paru nécessaire de comparer la situation des hommes et des femmes, ainsi que celle des mandataires sociaux et des administrateurs. Ces divers éléments sont abordés à la fois au niveau national des groupes et au niveau de trois caisses régionales coopératives.
* Centre de recherche sur l'intégration économique et financière (Crief; EA 2249), université de Poitiers.

** Centre interdisciplinaire d'analyse des processus humains et sociaux (CIAPHS; EA2241), université Rennes 2UEB.

*** Centre de recherche en gestion (Cerege; EA 1722), université de Poitiers.

Ce travail s'inscrit dans une recherche-action collective 2012-2014 sur le thème « La place et les spécificités des banques coopératives et mutualistes en Pays de Loire», portée par l'Observatoire régional de l'économie sociale et solidaire de la Cress Pays de Loire, en partenariat avec sept banques coopératives.

(1) Richez-Battesti N., Petrella F., Masonnasse J., Melnik K., "L'évaluation de la qualité de l'emploi au sein de l'ESS abordée par un faisceau d'indices ", in Caire G., Braconnier P., L'économie sociale et solidaire et le travail, L'Harmattan, 2013.

(2) Juridiquement, en France, la dénomination coopérative oumutualistepourlesbanques n'a aucune conséquence sur leur fonctionnement. Nous utilisons «banque coopérative » au sens général du terme. Nous employons "banque capitaliste ou commerciale» au sens de maximisation de la rentabilité du capital, 


\section{DE TRĖS FORTES DIFFÉRENCES SALARIALES AU SEIN DE L'ÉCONOMIE SOCIALE ET SOLIDAIRE}

En 2012, l'Institut national de la statistique et des études économiques (Insee) ${ }^{(3)}$ a souligné que les salaires, calculés sur une base annuelle en équivalent temps plein (ETP), sont en moyenne plus faibles dans l'ESS que dans le secteur privé (entendu hors ESS, donc commercial, market-based; $-16 \%$ ) et le secteur public (-7\%). La situation est cependant très différente entre, d'un côté, les associations (- $22 \%$ par rapport au secteur privé) et, de l'autre, les mutuelles et les coopératives (respectivement $+5 \%$ et $+7 \%$ ). L'étude relevait également que les écarts de salaires étaient en moyenne plus faibles dans l'ESS que dans le secteur privé. L'écart inter-décile entre les $10 \%$ les moins bien payés et les $10 \%$ les mieux payés était de 2,7, contre 3,1 dans le secteur privé. Cette contraction de l'échelle des salaires entre les moins bien lotis et les mieux lotis est conforme aux principes coopératifs de solidarité et d'équité.

Ce constat, fondé sur les différences statutaires, éclipse néanmoins de très forts contrastes de rémunérations entre secteurs d'activité. L'écart négatif au regard du privé est maximal pour les activités sportives, récréatives et de loisirs (- $44 \%)$, et l'écart positif le plus fort, pour le secteur de la santé (+ $28 \%$ ). Par ailleurs, une étude $\mathrm{Apec}^{(4)}$ de 2013 notait que les salaires proposés aux cadres dans les offres ESS étaient en moyenne plus faibles de $7 \%$ ( $-8 \%$ dans les associations, $-7 \%$ dans les mutuelles, mais $+11 \%$ dans les coopératives).

Qu'en est-il dans le secteur bancaire? L'étude Insee précitée proposait un court focus sur les banques coopératives. Le salaire annuel moyen brut (calculé en ETP) en 2009 était de 39200 euros dans les banques coopératives, contre 56630 euros dans les autres banques privées, soit un écart de $-30 \%$, en partie explicable par une proportion de cadres plus faible ( $23 \%$, contre $40 \%$ ). Il était également relevé que le salaire moyen (51640 euros) dans les filiales SA des banques coopératives était bien plus proche de celui des banques privées (un écart de seulement - $9 \%$ ), du fait d'une proportion de cadres plus élevée ( $37 \%)$. Ce sont ces éléments que nous allons approfondir.

\section{DES ÉCARTS SENSIBLES EN MATIĖRE DE RÉMUNÉRATION MOYENNE}

De façon générale, la rémunération mensuelle moyenne calculée sur l'ensemble des catégories de salariés dans les banques (3471 euros) est très largement supérieure à celle des salariés français (2005 euros; tableau 1). Le secteur de la banque (comme celui de l'assurance) est celui où les rémunérations se montrent les plus élevées. Les métiers liés à l’argent rémunèrent bien en général. Ce résultat est le produit de deux éléments: un effet de structure provenant d'une proportion de cadres beaucoup plus forte (45\%, contre $19 \%$ ) et des rémunérations plus élevées à la fois pour les non-cadres (2530 euros, contre 1645 euros) et pour les cadres (4494 euros, contre 3760 euros). 


\section{Tableau 1}

Rémunérations mensuelles moyennes des groupes bancaires (2012)

\begin{tabular}{|c|c|c|c|c|c|c|c|c|c|}
\hline & BPCE & $\begin{array}{l}\text { Crédit } \\
\text { agricole-LCL } \\
\text { (CA) }\end{array}$ & $\begin{array}{l}\text { Crédit } \\
\text { mutuel-CIC } \\
(\mathrm{CM})\end{array}$ & $\begin{array}{l}\text { Moyenne } \\
\text { banques } \\
\text { coopératives }\end{array}$ & $\begin{array}{l}\text { Société } \\
\text { générale } \\
\text { (SG) }\end{array}$ & $\begin{array}{l}\text { BNP Paribas } \\
\text { (BNPP) }\end{array}$ & $\begin{array}{l}\text { Moyenne } \\
\text { banques } \\
\text { capitalistes }\end{array}$ & $\begin{array}{l}\text { Moyenne } \\
\text { toutes } \\
\text { banques }\end{array}$ & $\begin{array}{l}\text { Tous salariés } \\
\text { France (2010)* }\end{array}$ \\
\hline $\begin{array}{l}\text { Nombre d'emplois } \\
\text { permanents } \\
\text { France }\end{array}$ & 71000 & 40000 & 62000 & 58000 & 41000 & 45000 & 43000 & 52000 & 24 millions \\
\hline $\begin{array}{l}\text { Proportion } \\
\text { de cadres }\end{array}$ & $34 \%$ & $56 \%$ & $41 \%$ & $42 \%$ & $51 \%$ & $53 \%$ & $52 \%$ & $45 \%$ & $19 \%$ \\
\hline $\begin{array}{l}\text { Rémunération } \\
\text { moyenne } \\
\text { des cadres }\end{array}$ & 3621 euros & 4733 euros & 4318 euros & 4128 euros & 6132 euros & 4407 euros & 5229 euros & 4494 euros & 3760 euros \\
\hline $\begin{array}{l}\text { Rémunération } \\
\text { moyenne } \\
\text { des non-cadres }\end{array}$ & 2536 euros & 2338 euros & 2630 euros & 2524 euros & 2602 euros & 2490 euros & 2543 euros & 2530 euros & 1645 euros \\
\hline $\begin{array}{l}\text { Rapport cadres- } \\
\text { non-cadres }\end{array}$ & 1,43 & 2,02 & 1,64 & 1,63 & 2,36 & 1,77 & 2,05 & 1,78 & 2,28 \\
\hline $\begin{array}{l}\text { Rémunération } \\
\text { totale moyenne }\end{array}$ & 2905 euros & 3575 euros & 3372 euros & 3227 euros & 4390 euros & 3572 euros & 3962 euros & 3471 euros & 2005 euros \\
\hline
\end{tabular}

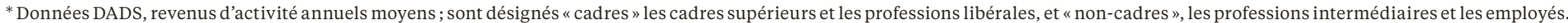
Sources de l'ensemble des tableaux et des graphiques : calculs des auteurs à partir des documents de référence et des bilans sociaux des banques. 
Cependant, la rémunération moyenne est significativement plus faible dans les banques coopératives (3227 euros) que dans les banques capitalistes (3962 euros), en raison d'une proportion moins importante de cadres ( $42 \%$, contre $52 \%$ ), qui sont en outre moins bien rémunérés (4128 euros, contre 5229 euros). Par contre, ce différentiel n'existe pas pour les non-cadres ( 2524 euros et 2543 euros). En conséquence, le ratio salarial entre cadres et non-cadres est plus faible dans les banques coopératives $(1,63)$ que dans les banques capitalistes $(2,05)$. Nous retrouvons là un indicateur objectif d'équité, déjà souligné précédemment sur le plan de l'ESS en général. Entre banques coopératives ${ }^{(5)}$, les écarts sont néanmoins sensibles. Le groupe Crédit agricole distribue une rémunération moyenne (3575 euros) identique à celle de BNP Paribas (3572 euros) et dispose d'un ratio de rémunérations cadres-non-cadres élevé $(2,02)$. Le groupe BPCE est celui où la rémunération moyenne est la plus faible des cinq groupes (2905 euros), compte tenu d'une proportion de cadres plus faible ( $34 \%$ ) et de leurs rémunérations plus basses (3621 euros).

Ces premiers constats sont toutefois à prendre avec prudence, car les bases de calcul des rémunérations moyennes calculées par chaque banque ne sont pas du tout harmonisées ${ }^{(6)}$.

Concernant les groupes coopératifs, il est également intéressant d'étudier ces mêmes données au niveau régional (tableau 2). Pour les deux caisses régionales dont nous avons pu obtenir le bilan social - l'une du Crédit agricole (CA-CR), l'autre du Crédit mutuel (CM-CR) -, la relative faible proportion de cadres ( $25 \%$ et $29 \%$ ) explique l'essentiel du différentiel au regard de l'ensemble du groupe. Les rémunérations moyennes sont similaires, tant pour les cadres (4362 euros et 4315 euros) que pour les non-cadres (2684 euros et 2568 euros). En revanche, la caisse régionale Banque populaire (dont nous n'avons que le rapport d'activité) se démarque nettement,
(5) Concernant la Société générale, dontletauxdecadre (51\%) est pourtant sensiblement du même niveau que celui de BNPP (53\%), le différentiel proviendrait d'une rémunération moyenne élevée pour les cadres (6132 euros), mais aussi sans doute en partie du fait que les données du bilan social semblent intégrer plus d'éléments de rémunération que les données proposées par les autres banques.

(6) BPCE : rémunération annuelle (sans précision); caisse régionale Banque populaire (BP-CR): salaire de base moyen; Crédit agricole (CA) Groupe: salaires mensuels moyens base brut; Crédit agricole SA (CASA): rémunération mensuelle moyenne fixe; caisse régionale de CA (CA-CR): masse salariale brute totale; Crédit mutuel $(\mathrm{CM})$ : rémunération annuelle moyenne brute; caisse régionale de $\mathrm{CM}(\mathrm{CM}$ $\mathrm{CR})$ : rémunération annuelle moyenne fixe; Société générale (SG): rémunération brute moyenneannuellecomprenant les éléments fixes et variables, les primes et les indemnités

\section{Tableau 2}

Rémunérations mensuelles moyennes dans les groupes coopératifs par rapport aux banques régionales (2012)

\begin{tabular}{|l|l|l|l|l|l|l|}
\hline & BPCE & BP-CR & CA & CA-CR & CM & CM-CR \\
\hline $\begin{array}{l}\text { Nombre d'emplois } \\
\text { permanents en France }\end{array}$ & 71000 & $\mathbf{1 5 0 0}$ & 40000 & $\mathbf{1 5 0 0}$ & 62000 & $\mathbf{2 0 0 0}$ \\
\hline $\begin{array}{l}\text { Proportion } \\
\text { de cadres }\end{array}$ & $34 \%$ & $\mathbf{3 3} \%$ & $56 \%$ & $\mathbf{2 5} \%$ & $41 \%$ & $\mathbf{2 9} \%$ \\
\hline $\begin{array}{l}\text { Rémunération } \\
\text { moyenne des cadres }\end{array}$ & 3621 euros & $\mathbf{4 0 4 2}$ euros & 4733 euros & $\mathbf{4 3 6 2}$ euros & 4318 euros & $\mathbf{4 3 1 5}$ euros \\
\hline $\begin{array}{l}\text { Rémunération moyenne } \\
\text { des non-cadres }\end{array}$ & 2536 euros & $\mathbf{2 4 0 2}$ euros & 2338 euros & $\mathbf{2 6 8 4}$ euros & 2630 euros & $\mathbf{2 5 6 8}$ euros \\
\hline $\begin{array}{l}\text { Rapport } \\
\text { cadres-non-cadres }\end{array}$ & 1,43 & $\mathbf{1 , 6 8}$ & 2,02 & $\mathbf{1 , 6 2}$ & 1,64 & $\mathbf{1 , 6 8}$ \\
\hline $\begin{array}{l}\text { Rémunération totale } \\
\text { moyenne }\end{array}$ & 2905 euros & $\mathbf{2 9 5 1 ~ e u r o s ~}$ & 3575 euros & $\mathbf{2 9 0 7}$ euros & 3372 euros & $\mathbf{3 2 5 0}$ euros \\
\hline
\end{tabular}


diverses (hors participation, intéressement et abondement); BNP Paribas (BNPP): rémunération mensuelle moyenne appointement fixe. (7) Ce résultat est à prendre avec précaution, car les pratiques de rémunérations à la Banque populaire et à la Caisse d'épargne diffèrent et ce constat étonnant.

(8) Rappelons que plus le coefficient de Gini est élevé et se rapproche de1, plusil indique une inégalité de répartition de la variable sous-jacente (ici les rémunérations). Les calculs ont été réalisés avec les répartitions des salariés en pourcentage, fournies dans les bilans sociaux des banques. Cette répartition ne repose pas systématiquement sur le même nombre d'échelons: détail fourni pour pour l'ensemble des salariés chez BNPP, 8 pour l'ensemble des salariés à la SG, 7 pour la caisse régionale de $\mathrm{CA}$ et 5 pour la caisse régionale sont peut-être les sources de 23 échelons chez CASA, 22

car la proportion de cadres et la rémunération moyenne sont identiques à celles qui existent au niveau national ${ }^{(7)}$.

\section{DES RÉMUNÉRATIONS VECTRICES D'INÉGALITÉS MOINS FORTES DANS LES BANQUES RÉGIONALES}

Afin de mieux spécifier les écarts de rémunération au sein de chaque banque, il est possible d'estimer les valeurs du coefficient de Gini ${ }^{(8)}$ sur la période 2010-2012, mais uniquement pour les quatre banques ${ }^{(9)}$ dont nous disposons des bilans sociaux: BNP Paribas, Société générale et les deux caisses régionales de Crédit mutuel et Crédit agricole. Nous y ajoutons CASA ${ }^{(10)}$, tête du groupe Crédit agricole, afin d'apprécier les écarts avec la caisse régionale du même groupe. En dépit de la diversité du nombre de salariés concernés, cette mesure est révélatrice de plusieurs éléments intéressants (graphique 1).

Tout d'abord, CASA et la Société générale apparaissent comme très inégalitaires (coefficients de Gini proches de 0,50). BNPP a une hiérarchie salariale plus ramassée (autour de 0,35).

Pour les caisses régionales, les valeurs sont nettement plus faibles $(0,25$ pour $C M-C R$ et 0,12 pour CA-CR) et indiquent une inégalité bien plus limitée qu'au niveau bancaire national et même plus faible que sur l'ensemble des salariés français, puisqu'en 2010 le coefficient de Gini (base: salaires mensuels nets équivalents temps complet; DADS) était de 0,28. Mis à part le plus faible nombre de hauts salaires, la différence que l'on peut souligner par rapport aux banques nationales réside dans des activités spéculatives nettement plus réduites et qui conduisent donc

\section{Graphique 1}

\section{Coefficient de Gini des rémunérations}

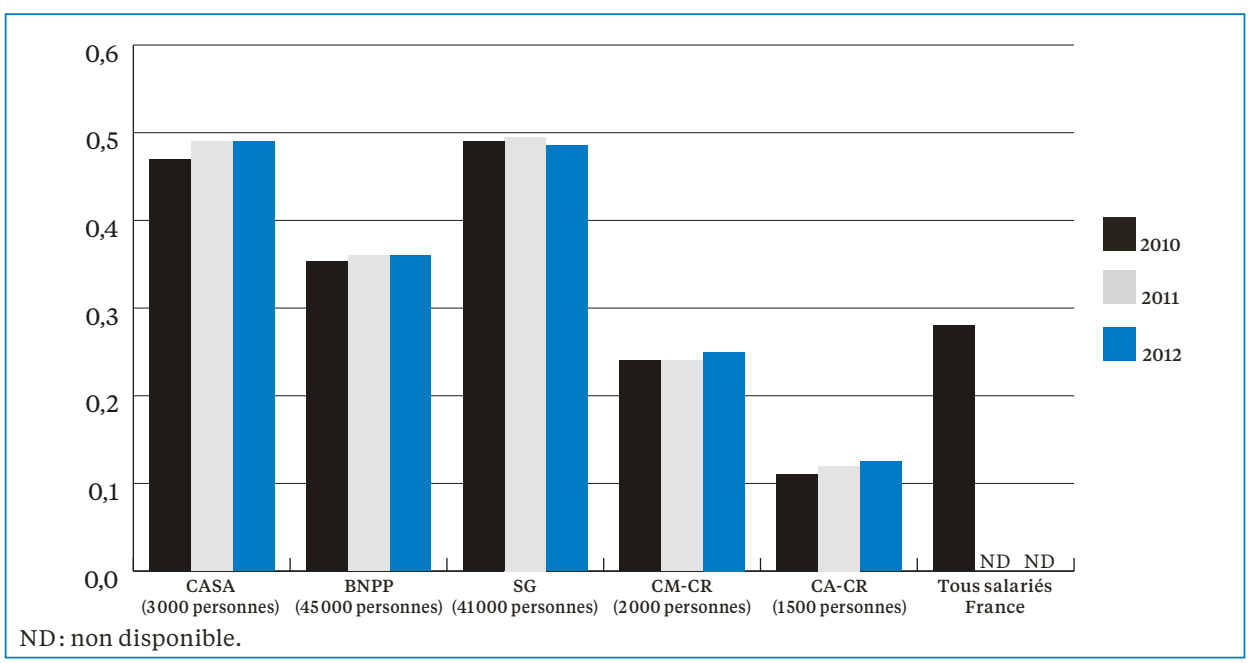


à des primes potentiellement moins fortes. En outre, le Crédit agricole et le Crédit mutuel Centre-Est Europe ${ }^{(11)}$, dont fait partie CM-CR, ont chacun une convention collective propre différente de la convention collective nationale de la banque. Sur le moyen terme, si l'on tient compte de l'évolution de la situation sur les trois années, les valeurs se révèlent stables pour chaque banque considérée.

Regardons à présent ce qu'il en est du rapport des rémunérations entre les $10 \%$ des salariés les mieux payés et les $10 \%$ les moins bien payés (graphique 2). Confirmant les constats sur les coefficients de Gini, les écarts au sein d'une même banque vont de 2,5 à 3 pour les deux banques régionales considérées et à 8 pour la SG en 2011, et sur les trois années analysées ces écarts entre hauts et bas salaires évoluent assez peu. La SG affiche les écarts les plus marqués, suivie par CASA (avec beaucoup moins de salariés) et BNPP. Un effet de la taille de la banque est ici indéniable, avec davantage d'amplitude salariale pour les grosses structures.

Nous pouvons compléter les résultats précédents avec le montant global des dix rémunérations les plus élevées (tableau 3 , en page suivante). Sans surprise, les banques régionales rémunèrent leurs dirigeants avec beaucoup plus de modération que ne le font les banques nationales (BNPP et SG notamment), dont on remarque également la baisse des hautes rémunérations sur l'année 2012. Les écarts vont de 1 à 15 entre la caisse régionale du Crédit mutuel et la SG. Ces chiffres confirment la tendance, relevée précédemment, à de moins fortes inégalités salariales dans les caisses régionales. de CM. Ces différences ne portent pas à conséquence sur les résultats et les conclusions dans la mesure où les écarts entre banques sont suffisamment nets.

(9) Il nous a été impossible de disposer des bilans sociaux des trois groupes coopératifs. (10) Au profil particulier: $90 \%$ de cadres, dont la rémunération mensuelle moyenne est de 5390 euros. La comparaison de CASA avec les autres banques doit être considérée avec précaution pour trois raisons importantes: la taille de la structure (3000 personnes, contre plus de 40000 pour la SG et BNPP); le type d'activité (organe de " tête » du groupe Crédit agricole sans activité locale); et la forme juridique (CASA est une entreprise société anonyme (SA) et est cotée, contrairement aux banques régionales).

(11) La convention collective ne s'applique toutefois pas à certaines filiales de CASA(LCL ou CA-CIB, banque de finan-

\section{Graphique 2}

Ratio $10 \%$ des salariés les mieux payés par rapport aux $10 \%$ les moins bien payés

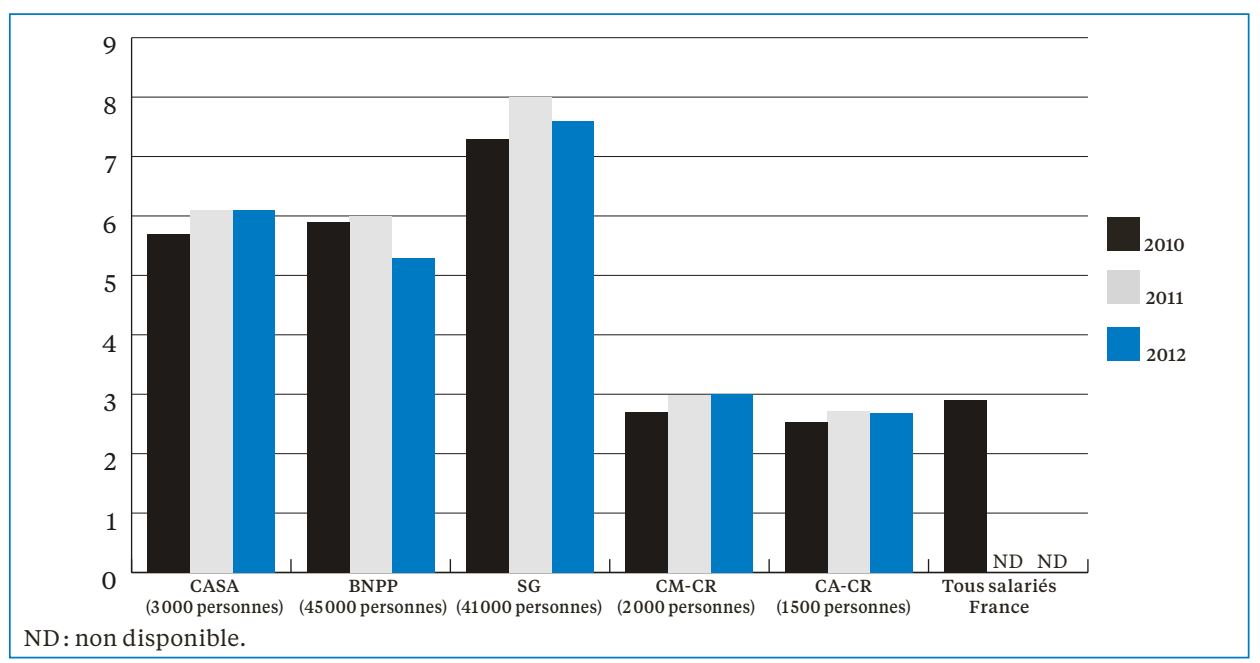


cement et d'investissement du groupe CA, par exemple) ou du CM11 - regroupant CIC, Crédit mutuel Centre-Est Europe et toutes les entités qui en dépendent - (CIC, par exemple), qui relèvent de la convention collective nationale de la banque.

\section{DES COMPLÉMENTS DE RÉMUNÉRATION COMPARABLES ENTRE LES DEUX TYPES DE BANQUES (SAUF CASA)}

Le poids de la participation et de l'intéressement varie fortement en proportion des charges de personnel suivant les banques et le nombre de salariés (le total en France ou la société mère uniquement), ainsi naturellement que suivant les années, nettement bénéficiaires ou non. CASA (société mère) verse une participation et un intéressement particulièrement élevés au regard de ses charges de personnel $(9,02 \%$ et 7,00 \% en 2012) comparé à BNPP (respectivement $2,56 \%$ et $2,57 \%$ ) ou à la SG (respectivement 0,10 \% et 0,62 \%). La chute enregistrée en 2012 par rapport aux années antérieures est commune à toutes les banques, mais dans une amplitude variable.

Les montants moyens par salarié (tableau 5 , en page suivante) confirment les indications du tableau en pourcentage (tableau 4): les rémunérations complémentaires CASA sont particulièrement élevées, ce qui est logique

\section{Tableau 3}

Montant moyen des dix rémunérations annuelles les plus élevées

\begin{tabular}{|l|l|l|l|}
\hline & $\mathbf{2 0 1 0}$ & $\mathbf{2 0 1 1}$ & $\mathbf{2 0 1 2}$ \\
\hline CASA & 1403655 euros & 1096440 euros & 828473 euros \\
\hline BNPP & 1789670 euros & 1890023 euros & 1450662 euros \\
\hline SG & 2064200 euros & 2454900 euros & 1913300 euros \\
\hline CM-CR & 126302 euros & 133626 euros & 133890 euros \\
\hline CA-CR & 171946 euros & 168788 euros & 163652 euros \\
\hline
\end{tabular}

\section{Tableau 4}

\section{Rémunérations complémentaires en pourcentage}

\begin{tabular}{|l|l|l|c|}
\hline Rémunérations en pourcentage des charges de personnel totales & 2010 & 2011 & 2012 \\
\hline BNP Paribas (BNPP) & & & \\
\hline Réserve spéciale de participation & $3,19 \%$ & $2,30 \%$ & $2,56 \%$ \\
\hline Intéressement & $3,76 \%$ & $3,30 \%$ & $2,57 \%$ \\
\hline Abondement versé par BNPP dans le cadre de l'épargne salariale & $1,49 \%$ & $1,35 \%$ & $1,29 \%$ \\
\hline Crédit agricole SA (CASA) & & & \\
\hline Réserve spéciale de participation & $10,20 \%$ & $12,29 \%$ & $9,02 \%$ \\
\hline Intéressement & $9,00 \%$ & $10,60 \%$ & $7,00 \%$ \\
\hline Abondement versé par CASA dans le cadre de l'épargne salariale & $0,76 \%$ & $1,26 \%$ & $1,42 \%$ \\
\hline Société générale (SG) & & & \\
\hline Réserve spéciale de participation & $0,25 \%$ & $0,27 \%$ & $0,10 \%$ \\
\hline Intéressement & $0,87 \%$ & $0,77 \%$ & $0,62 \%$ \\
\hline Abondement versé par la SG dans le cadre de l'épargne salariale & $0,63 \%$ & $0,68 \%$ & $0,42 \%$ \\
\hline
\end{tabular}


pour une société mère composée à $90 \%$ de cadres, qui plus est de haut niveau. Il faut également noter que les salariés des deux caisses régionales disposent de rémunérations complémentaires de niveau comparable, voire supérieur à ceux des deux groupes capitalistes.

\section{UNE ÉGALITÉ FEMMES-HOMMES ENCORE À VENIR}

Lorsque l'on s'intéresse à l'égalité des rémunérations entre les femmes et les hommes (tableau 6, en page suivante), le premier constat - bien connu est que la profession bancaire est davantage féminisée par rapport à la moyenne de la population active. Il semble que cette féminisation soit très légèrement plus faible dans les banques coopératives que dans les banques capitalistes, l'exception étant CASA, du fait du poids considérable

\section{Tableau 5}

\section{Rémunérations complémentaires en euros}

\begin{tabular}{|c|c|c|c|}
\hline Rémunérations moyennes en euros & 2010 & 2011 & 2012 \\
\hline \multicolumn{4}{|l|}{ BNP Paribas } \\
\hline Participation & 2470 euros & 1863 euros & 2035 euros \\
\hline Intéressement & 2913 euros & 2673 euros & 2049 euros \\
\hline Abondement versé dans le cadre de l'épargne salariale & 1156 euros & 1096 euros & 1024 euros \\
\hline Total & 6539 euros & 5633 euros & 5108 euros \\
\hline \multicolumn{4}{|l|}{ Crédit agricole SA (CASA) } \\
\hline Participation & 7071 euros & 9017 euros & 6048 euros \\
\hline Intéressement & 6240 euros & 7779 euros & 4693 euros \\
\hline Abondement versé dans le cadre de l'épargne salariale & 526 euros & 924 euros & 953 euros \\
\hline Total & 13838 euros & 17719 euros & 11694 euros \\
\hline \multicolumn{4}{|l|}{ Société générale (SG) } \\
\hline Participation & 572 euros & 620 euros & 241 euros \\
\hline Intéressement & 2004 euros & 1758 euros & 1435 euros \\
\hline Abondement versé dans le cadre de l'épargne salariale & 1449 euros & 1565 euros & 975 euros \\
\hline Total & 4026 euros & 3943 euros & 2650 euros \\
\hline \multicolumn{4}{|l|}{ Crédit mutuel caisse régionale (CM-CR) } \\
\hline Participation & $\mathrm{nc}$ & 1422 euros & 1416 euros \\
\hline Intéressement & 5758 euros & 5004 euros & 2979 euros \\
\hline Abondement versé dans le cadre de l'épargne salariale & $\mathrm{nc}$ & $\mathrm{nc}$ & $\mathrm{nc}$ \\
\hline Total & nc & 6426 euros & 4395 euros \\
\hline \multicolumn{4}{|l|}{ Crédit agricole caisse régionale (CA-CR) } \\
\hline Participation & 1353 euros & 833 euros & 524 euros \\
\hline Intéressement & 4064 euros & 4720 euros & 4743 euros \\
\hline Abondement versé dans le cadre de l'épargne salariale & $\mathrm{nc}$ & nc & $\mathrm{nc}$ \\
\hline Total & 5417 euros & 5553 euros & 5267 euros \\
\hline
\end{tabular}


(12) Là encore, les données proposées par les bilans sociaux ou les documents de référence ne sont pas harmonisées. Le tableau 6 est construit sur les bases suivantes: BPCE: CDI; BP-CR: CDI; CA Groupe: salaires mensuels moyens des effectifs CDI actifs (salaire brut de base); CASA: rémunérations du mois de décembre, hors primes, del'effectif payédouze mois et dont le taux d'activité est redressé à $100 \%$; CA-CR: salaire brut fiscal (SBF) CDI à temps plein; $\mathrm{CM}$ : non disponible; $\mathrm{CM}-\mathrm{CR}$ : rémunération annuelle moyenne (effectif permanent - brut global); SG : tout type; BNPP: rémunération mensuelle moyenne appointement fixe. des cadres dans l'effectif global (90\%). Deuxième constat - là encore, bien connu -, la proportion de femmes est systématiquement plus faible chez les cadres que chez les non-cadres. Et l'ensemble des banques confirme ce résultat, avec un taux de féminisation des cadres particulièrement faible dans les groupes BPCE (36\%) et Crédit mutuel (34\%). Il faut également noter que dans les trois groupes coopératifs cette donnée est systématiquement plus faible au niveau de la caisse régionale qu'au niveau du groupe. En termes de rémunérations ${ }^{(12)}$ sur l'effectif global, la Société générale est très largement le groupe le plus égalitaire ( $93 \%)$, le groupe CA arrivant dernier (74\%), pour un ratio toutes entreprises françaises de $81 \%$. Cependant, là aussi, il est nécessaire de distinguer non-cadres et cadres. Pour les premiers, les écarts de rémunération sont faibles, voire même dans certains cas très légèrement favorables aux femmes. Pour les seconds, les écarts sont plus importants ( $9 \%$ à BPCE et $18 \%$ dans le groupe CA), mais moins forts qu'au niveau de l'ensemble des entreprises (22\%).

L'égalité salariale, mais aussi de statut, entre femmes et hommes est donc loin d'être atteinte, et les banques coopératives ne se distinguent pas des deux groupes capitalistes sur ce point. Pourtant, à la lecture de leur bilan social ou de leur rapport RSE,

\section{Tableau 6}

Proportions femmes-hommes et ratios de rémunération

\begin{tabular}{|l|l|l|l|l|l|l|l|l|l|l|}
\hline 2012 & BPCE & BP-CR & $\begin{array}{l}\text { CA } \\
\text { Groupe }\end{array}$ & CASA & CA-CR & CM & CM-CR & SG & BNPP & $\begin{array}{l}\text { France, } \\
\text { toutes } \\
\text { entreprises }\end{array}$ \\
\hline $\begin{array}{l}\text { Proportion } \\
\text { de femmes } \\
\text { dans l'effectif }\end{array}$ & $55 \%$ & $53 \%$ & $57 \%$ & $48 \%$ & $56 \%$ & $55 \%$ & $56 \%$ & $56 \%$ & $58 \%$ & $52 \%$ \\
\hline $\begin{array}{l}\text { Proportion } \\
\text { de femmes } \\
\text { chez les cadres }\end{array}$ & $36 \%$ & $29 \%$ & $46 \%$ & $45 \%$ & $36 \%$ & $34 \%$ & $++30 \%$ & $43 \%$ & $46 \%$ & $45 \% *$ \\
\hline $\begin{array}{l}\text { Proportion } \\
\text { de femmes chez } \\
\text { les non-cadres }\end{array}$ & $65 \%$ & $65 \%$ & $72 \%$ & $75 \%$ & $63 \%$ & $70 \%$ & $68 \%$ & $70 \%$ & $71 \%$ & $\begin{array}{l}57 \% * * \\
82 \% * *\end{array}$ \\
\hline $\begin{array}{l}\text { Ratio rémunération } \\
\text { femmes-hommes } \\
\text { total }\end{array}$ & $82 \%$ & $77 \%$ & $74 \%$ & $80 \%$ & ND & ND & $78 \%$ & $93 \%$ & $79 \%$ & $81 \%$ \\
\hline $\begin{array}{l}\text { Ratio rémunération } \\
\text { femmes-hommes } \\
\text { cadres }\end{array}$ & $91 \%$ & $87 \%$ & $82 \%$ & $83 \%$ & ND & ND & $88 \%$ & $87 \%$ & $88 \%$ & $78 \% *$ \\
\hline $\begin{array}{l}\text { Ratio rémunération } \\
\text { femmes-hommes } \\
\text { non-cadres }\end{array}$ & $94 \%$ & $94 \%$ & $101 \%$ & $101 \%$ & ND & ND & $95 \%$ & $101 \%$ & $98 \%$ & $\begin{array}{l}87 \% * * \\
92 \% * *\end{array}$ \\
\hline
\end{tabular}

* Cadres administratifs et commerciaux d'entreprise.

** Professions intermédiaires administratives et commerciales des entreprises.

*** Employés administratifs d'entreprise. 
les banques coopératives ont davantage tendance à mettre en exergue leur valorisation de la diversité et de la parité parmi leurs salariés ${ }^{(13)}$.

\section{RÉMUNÉRATION DES MANDATAIRES SOCIAUX ET INDEMNITÉS DES ADMINISTRATEURS}

Au niveau national, les présidents et les directeurs généraux des banques coopératives disposent de rémunérations d'un niveau absolu plus faible que celles qui sont délivrées dans les banques capitalistes (tableau 7). Cependant, elles restent particulièrement élevées: 600000 euros pour les présidents, 1 million d'euros pour les directeurs généraux. Par ailleurs, le « coût » (jetons de présence) d'un conseil d'administration national de banque coopérative est supérieur à celui des banques capitalistes.

Trois remarques peuvent être faites:

- le principe d'économie sociale de gestion à caractère désintéressé des dirigeants élus, président et administrateurs, n’est pas respecté;

- à titre de comparaison, le gouvernement français a décidé par un décret du 25 juillet 2012 qu'un dirigeant de société publique ne pouvait pas disposer d'une rémunération annuelle supérieure à 450000 euros;

- s'agissant des caisses régionales, les présidents de conseils d'administration sont en général très confortablement indemnisés. Ainsi, les présidents des dix-sept caisses d'épargne ont une rémunération, part fixe plus part variable, qui se situe entre 391000 et 691000 euros.

\section{CONCLUSION}

Sous l'angle des rémunérations, les banques coopératives semblent finalement en moyenne plus responsables et raisonnables que les banques capitalistes, notamment au niveau des caisses régionales. Mais les écarts entre groupes bancaires coopératifs restent importants. En quelque sorte, plus un groupe bancaire coopératif repose sur un modèle hybride alliant coopération et capitalisme, comme c'est le cas du Crédit agricole, plus les comportements en termes de salaires convergent vers ceux des établissements bancaires commerciaux (privés lucratifs).

\section{Tableau 7}

«Coût » de la dirigeance

\begin{tabular}{|l|l|l|l|}
\hline 2012 & $\begin{array}{l}\text { Moyenne CA + CM + } \\
\text { BPCE }\end{array}$ & Moyenne BNP + SG & Ecart \\
\hline Président du conseil d'administration & 579725 euros & 1696974 euros & 1 à 3 \\
\hline Directeur général & 1096415 euros & 2638324 euros & 1 à 2,4 \\
\hline Total jetons de présence du CA & 1257284 euros* & 1032498 euros & $+21 \%$ \\
\hline
\end{tabular}

* Calcul pour CA et BPCE. Le rapport du Crédit mutuel est peu précis sur ce point.
(13) Le Crédit mutuel, par exemple, indique dans son port RSE de 2013 (p. 27): Plusieurs groupes régionaux pourla parité, mises en œuvre notamment dans le cadre des recrutements et desévolutions e carrière.» 
Par ailleurs, il reste des progrès à faire pour certains groupes bancaires coopératifs qui cherchent à obtenir le «label Egalité », témoignant de la volonté d'améliorer l'égalité professionnelle entre hommes et femmes. Allant plus loin, l'un des responsables d'une caisse régionale étudiée affirme: "Ce label Egalité récompense notre engagement à promouvoir l'égalité hommes-femmes chez nos collaborateurs. Mais notre réflexion porte aussi sur la place des femmes dans nos conseils d'administration et la façon de les faire progresser. » Et en ce domaine, le pas à franchir est particulièrement important. Les conseils d'administration des structures faitières du Crédit agricole et de BPCE, avec $28 \%$ de femmes, sont en dessous de la BNP (37\%) et de la Société générale (40\%). Les « instances politiques », les fédérations nationales, sont encore moins féminisées : $9 \%$ de femmes à la Fédération nationale des banques populaires (FNBP), $12 \%$ à la Fédération nationale des caisses d'épargne (FNCE; dont la directrice générale est toutefois une femme) et $0 \%$ à la Fédération nationale du Crédit agricole (FNCA). Le Crédit mutuel quant à lui est, sur le plan national, quasi exclusivement dirigé par des hommes.

Enfin, les rémunérations restent aussi interrogeables au regard des valeurs et des principes de l'économie sociale. Lors d'une assemblée générale de CASA, en réponse à la question d'un actionnaire s'étonnant du niveau de rémunération des hauts dirigeants de cette banque coopérative, le directeur général de l'époque avait justifié la situation ainsi : «Pour jouer en première division, il faut des salaires de première division. » Pour filer cette métaphore footballistique, il est en effet indéniable que les groupes bancaires coopératifs français jouent bien "en première division »: mesuré en total d'actifs, le Crédit agricole est la troisième banque européenne, BPCE est au dixième rang et le Crédit mutuel à la vingtième place (données de 2012). Concernant les rémunérations, elles sont également bien de "première division ", au regard de celles qui sont pratiquées dans l'immense majorité de l'ESS. Certes, les écarts de rémunération - sauf sur l'aspect femmes-hommes et les très hauts salaires sont sensiblement plus raisonnables

(14) Sur le plan européen, en total d'actifs, BNP Paribas est quatrième, et la Société générale, huitième. que dans les banques capitalistes de "première division» ${ }^{(14)}$. Néanmoins, aucune des banques coopératives étudiées ne remet réellement en cause cette posture de raisonnement, symbolique d'un isomorphisme organisationnel croissant.

\section{A RETROUVER DANS LES ARCHIVES DE LA «RECMA » (RECMA.ORG/ARTICLES)}

Marsal C., «Quelles sont les spécificités du management des banques coopératives ? », Recma, $\mathrm{n}^{\circ} 322,2011$.

Kontoyannatos C., « Les banques coopératives en Grèce : un développement au service des membres », Recma, $\mathrm{n}^{\circ}$ 280, 2001.

Parodi M., «Les banques coopératives dans le jeu capitaliste mondial », Recma, n² 277, 2000. 